\title{
Application of Object-Oriented Mathematical Modeling and Computer Algebra in Mathematical Economic Analysis
}

\author{
Dr. R. Seenivasan ${ }^{*}$
}

Assistant Professor, Department of Mathematical Economics, School of Economics, M. K. University, Palkalai Nagar, Tamil Nadu 625021, India

DOI: $10.36348 / \mathrm{sb} .2020 . v 06 i 04.003$

| Received: 14.01.2020 | Accepted: 21.01.2020 | Published: 30.04.2020

*Corresponding author: Dr. R. Seenivasan

\section{Abstract}

This research paper, we present the first programming environment and modeling language which integrates inheritance within a computer algebra language. This environment and language, called Object Math (Object oriented Mathematical language for scientific computing), is currently being used for industrial applications in advanced mathematical economic analysis, but is generally applicable to other areas. Using Object Math, it is possible to model classes of equation objects, to support inheritance of equations, and to solve systems of equations. The Object Math environment is designed to handle realistic problems. This is achieved by allowing the user to specify transformations and simplifications of formulae in the model, in order to arrive at a representation which is efficiently solvable. Such algebraic transformations can conveniently be supported since Object Math models are translated into the Mathematica computer algebra language. When necessary, equations can be transformed to $\mathrm{C}++$ code for effic.

Keywords: Modeling diagram, Mathematical Economic analysis, Mathematical modeling, Computer Algebra, Model classes, Formula, Algebra language, Equations.

Copyright @ 2020: This is an open-access article distributed under the terms of the Creative Commons Attribution license which permits unrestricted use, distribution, and reproduction in any medium for non-commercial use (NonCommercial, or CC-BY-NC) provided the original author and source are credited.

\section{INTRODUCTION}

In the last ten years the pattern of the prior sixty years rapid growth in both the theory and range of uses for applied and applicable mathematics has continued, and accelerated. Examples of situations where applied mathematics has had important influence and where new applications are emerging rapidly include:

- Cell phones and global positioning systems

- Medical imaging

- Weather and climate modeling

- Financial mathematics

- Mechanism design, market design, and auctions

- Web searching, routing, and sensor networks

- Extracting information, such as genome data, from large data sets.

These areas have drawn on mathematical tools from both classical applied mathematics and discrete applied methodologies. The tremendous increase in storage and speed of computers has revitalized many areas of applied mathematics because, with computer power now available, problems can be pushed towards solutions in ways that were formerly impossible.

\section{Mathematical formulation}

This is usually the trickiest part of the modeling process, requiring the choice of a mathematical structure, which is appropriate for the model objectives and is able to describe in quantitative form the hypotheses. This step of the process requires a certain level of mathematical sophistication and, more importantly, it requires to define vague concepts and loose relations in strict mathematical terms. Noticeably, model objectives play an important role because, a detailed description of the biological system may turn out to be useless if not required by the model objectives.

Choosing mathematical formulations is a mapping of the model into the mathematical domain to obtain a formal model. A good formal model must be a compromise between the competing properties of any model (Realism, Precision and Generality) and should take into account some specificity of the mathematical domain. Accordingly, we can identify the major 
properties of formal models as follows: 'relevance', capturing the essential properties of the phenomenon; 'computability', transfer model hypothesis into a mathematical / computational infrastructure that can be solved to give the desired results with the required precision; 'understandability', offering a conceptual framework for thinking about the scientific domain; 'extendibility', allowing the inclusion of additional realworld objects in the same mathematical scheme. Taking into account the biological complexity, a very detailed model of a biological system may work out to be unsuitable to automatic resolution, i.e. not computable (for instance, too many equations to be solved or too many unknown parameters to be estimated); in contrast, a too simplistic model may not be able to account for the complexity of the biological system of interest.

A good formal model must be understandable by domain experts so that they could use it for their own quantitative reasoning. Finally, as biology is a fast growing science, extensibility is an important characteristic for biological models. When new objects and relations in the system are derived from laboratory experiments, it should be easy to extend the model with minor changes in the mathematical structure.

In most cases models are equipped with just one mathematical structure of those mentioned previously. However, there are also 'hybrid' models where different mathematical structures are used in combination but also models which add to a detailed description of the system of interest other types of models (e.g. BBM), which mimic the effects of other systems interlinked with the one under investigations. We will describe some hybrid models in the section 'Tools and Applications'.

\section{Mathematical modeling and applied mathematics}

Mathematical modeling might be described as using mathematics "outside of mathematics," in "realworld situations." Mathematical modeling has become even more important since the newly adopted Common Core State Standards in Mathematics include a Standard for Mathematical Practice which stipulates that students be able to "model with mathematics." While there is a tradition of "story problems" in mathematics, many are silly (A collection of cows and chickens includes 28 legs. I see 9 heads. How many ... .). Since many mathematics majors plan on K-12 teaching, it is important that courses in the major not only treat applied mathematics but in general but also present mathematical modeling in ways that help future teachers. Some mathematical modeling courses may serve both students who plan K-6 teaching and also students seeking general education requirements. Some institutions (especially two year colleges) are substituting Mathematical Modeling courses for College Algebra, hoping that such courses will better serve students' needs and perhaps encourage some to consider majoring in STEM disciplines.

\section{Different views of mathematics}

As a complex and multilayered subject, mathematics can be viewed from many vantage points. One view of mathematics stresses attempts to solve specific problems; a second emphasizes theoretical frameworks. Many people are attracted to mathematics through competitions and wide-ranging problems; some distinguished mathematicians who have reputations as problem solvers. Others are known for theoretical exploration, over long periods of time, of particular pieces of the mathematical landscape. Mathematical modeling can illuminate the connection between problem solving and theoretical mathematics. Teaching modeling helps students develop of skill in attacking applied questions; situations to be modeled play the same role in applied mathematics as theoretical problems play in theoretical mathematics.

\section{Majors, minors, and tracks in applied mathematics}

Departments will naturally strive to serve the needs of all majors in theoretical and applied mathematics. One possibility is to offer applied mathematics majors, minor or tracks in specific areas (e.g., mathematical physics, economics, biology, ecology, actuarial science, network science and bioinformatics). Many departments design their majors with a relatively small set of "core" required courses; students then select one of several applied-flavor tracks, each with its own required and elective courses, to complete the major.

\section{A list of applications}

In the following, I give a list of applications whose modeling I understand, at least in some detail. All areas mentioned have numerous mathematical economic challenges. This list is based on my own experience; therefore it is very incomplete as a list of applications of mathematical economics in general. There are an almost endless number of other areas with interesting mathematical economic problems. Indeed, mathematical economics is simply the language for posing problems precisely and unambiguously (so that even a stupid, pedantic computer can understand it).

\section{Anthropological Economics \\ - Modeling, classifying and reconstructing skulls}

\section{Archeological Economics \\ - Reconstruction of objects from preserved fragments \\ - Classifying ancient artifices}

\section{Architecture \\ - Virtual reality}


Artificial intelligence economy

- Computer vision

- Image interpretation

- Robotics

- Speech recognition

- Optical character recognition

- $\quad$ Reasoning under uncertainty

Arts

- Computer animation (Jurassic Park)

\section{Astronomy}

- Detection of planetary systems

- Correcting the Hubble telescope

- Origin of the universe

- Evolution of stars

\section{Bio - Economics}

- $\quad$ Protein folding

- Human genome project

- Population dynamics

- Morphogenesis

- Evolutionary pedigrees

- $\quad$ Spreading of infectious diseases (AIDS)

- Animal and plant breeding (genetic variability)

\section{Chemical engineering economics}

- Chemical equilibrium

- $\quad$ Planning of production units

\section{Chemistry}

- Chemical reaction dynamics

- Molecular modeling

- Electronic structure calculations

\section{Computer applications in Economics}

- Image processing

- Realistic computer graphics (ray tracing)

\section{Criminalistic science}

- Finger print recognition

- Face recognition

\section{Mathematical Economics}

- Labour data analysis

\section{Engineering Economics}

- Stability of electric circuits

- Microchip analysis

- Power supply network optimization

\section{Financial Economics}

- Risk analysis

- Value estimation of options

Fluid mechanic economy

- Wind channel
- Turbulence

\section{Geosciences economy}

- Prediction of oil or ore deposits

- Map production

- Earth quake prediction

\section{Internet Economics}

- Web search

- Optimal routing

Linguistics economy

- Automatic translation

Materials Science

- Microchip production

- Microstructures

- Semiconductor modeling

Mechanical engineering economics

- Stability of structures (high rise buildings, bridges, air planes)

- Structural optimization

- Crash simulation

Medical Economics

- Radiation therapy planning

- Computer-aided tomography

- Blood circulation models

Meteorology

- Weather prediction

- Climate prediction (global warming, what caused the ozone hole?)

Music Economics

- Analysis and synthesis of sounds

Neuroscience

- Neural networks

- Signal transmission in nerves

Pharmacology

- Docking of molecules to proteins

- Screening of new compounds

Physical Economics

- Elementary particle tracking

- Quantum field theory predictions (baryon spectrum)

- Laser dynamics

Political Sciences

- Analysis of elections

Psychological Economics

- Formalizing diaries of therapy sessions 
Space Sciences

- Trajectory planning

- Flight simulation

- Shuttle reentry

\section{Transport Economics}

- Air traffic scheduling

- Taxi for handicapped people

- Automatic pilot for cars and airplanes

\section{Basic Numerical Tasks}

The following is a list of categories containing the basic algorithmic toolkit needed for extracting numerical information from mathematical economic models. Due to the breadth of the subject, this cannot be covered in a single course. For a thorough education one needs to attend courses (or read books) at least on numerical analysis (which usually covers some numerical linear algebra, too), optimization, and numerical methods for partial differential equations. Unfortunately, there appear to be few good courses and books on (higher-dimensional) numerical data analysis.

\section{Numerical linear algebra}

- Linear systems of equations

- Eigen value problems

- Linear programming (linear optimization)

- Techniques for large, sparse problems

\section{Numerical analysis}

- Function evaluation

- Automatic and numerical differentiation

- Interpolation

- Approximation (Padé, least squares, radial basis functions)

- Integration (univariate, multivariate, Fourier transform)

- Special functions

- Nonlinear systems of equations

- $\quad$ Optimization = nonlinear programming

- Techniques for large, sparse problems

\section{Numerical data analysis (= numerical statistics)}

- Visualization (2D and 3D computational geometry)

- Parameter estimation (least squares, maximum likelihood)
- Prediction

- Classification

- Time series analysis (signal processing, filtering, time correlations, spectral analysis)

- Categorical time series (hidden Markov models)

- Random numbers and Monte Carlo methods

- Techniques for large, sparse problems

\section{Numerical functional analysis}

- Ordinary differential equations (initial value problems, boundary value problems, eigen value problems, stability)

- Techniques for large problems

- Partial differential equations (finite differences, finite elements, boundary elements, mesh generation, adaptive meshes)

- Stochastic differential equations

- Integral equations (and regularization)

\section{Non-numerical algorithms}

- Symbolic methods (computer algebra)

- Sorting

- Compression

- Cryptography

- Error correcting codes

\section{Modeling diagram}

Mathematical modeling

- $\quad$ is indispensable in many applications

- is successful in many further applications

- gives precision and direction for problem solution

- enables a thorough understanding of the system modeled

- $\quad$ prepares the way for better design or control of a system

- $\quad$ allows the efficient use of modern computing capabilities

Learning about mathematical modeling is an important step from a theoretical mathematical training to an application-oriented mathematical expertise, and makes the student fit for mastering the challenges of our modern technological culture. The nodes of the following diagram represent information to be collected, sorted, evaluated, and organized. 


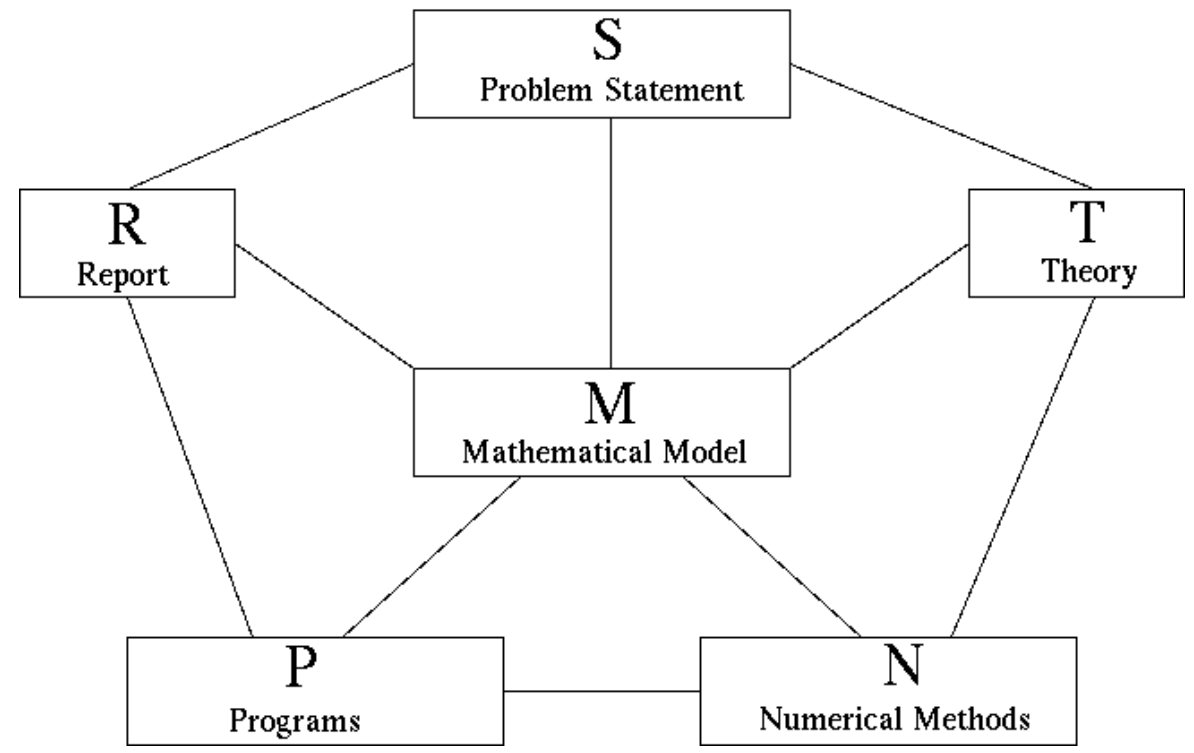

The edges of the diagram represent activities of two-way communication (flow of relevant information) between the nodes and the corresponding sources of information.

\section{S. Problem Statement}

- Interests of customer/boss

- Often ambiguous/incomplete

- Wishes are sometimes incompatible

\section{Mathematical Model}

- Concepts / Variables

- Relations

- Restrictions

- Goals

- Priorities / Quality assignments

\section{T. Theory}

- of Application

- of Mathematics

- Literature search

\section{N. Numerical Methods}

- Software libraries

- Free software from WWW

- Background information

\section{P. Programs}

- Flow diagrams

- Implementation

- User interface

- Documentation

\section{R. Report}

- Description

- Analysis

- Results

- Validation
- Visualization

- Limitations

- Recommendations

From mathematical formulation to the numerical solution

Only very simple models can be analyzed analytically (i.e. by algebraic derivation of the system properties). In most cases the model is either directly implemented as a computer code (i.e. the algorithm-like in $\mathrm{ABM}$ ) or equations must be solved numerically. Even if there are a variety of methods for solving equations, transferring equations into computer code is a possible source of error and appropriate method to avoid errors due to numerical instabilities need to be carefully chosen. Models of biological systems can involve scores of dynamic variables and thousands of parameters, especially when spatial processes are investigated. In this respect, checking the computer results to match available data is not a trivial exercise.

\section{Using the modeling diagram}

- The modeling diagram breaks the modeling task into $16=6+10$ different processes.

- Each of the 6 nodes and each of the 10 edges deserve repeated attention, usually at every stage of the modeling process.

- The modeling is complete only when the 'traffic' along all edges becomes insignificant.

- Generally, working on an edge enriches both participating nodes.

- If stuck along one edge, move to another one! Use the general rules below as a check list!

- Frequently, the problem changes during modeling, in the light of the understanding gained by the modeling process. At the end, even a vague or contradictory initial problem description should have developed into a reasonably well-defined description, with an 
associated precisely defined (though perhaps inaccurate) mathematical model.

\section{Population dynamics}

In another study, the authors describe and analyze a periodically forced difference equation model for malaria in mosquitoes that captures the effects of seasonality and allows the mosquitoes to feed on a heterogeneous population of hosts. With the integration of the difference equation model with an individualbased stochastic simulation model for malaria in humans, they compare the effects of insecticide-treated nets (ITNs) and indoor residual spraying (IRS) in reducing malaria transmission, prevalence and incidence. They conclude showing that ITNs are more effective than IRS in reducing transmission and prevalence, proving also that the combination of both interventions is more effective than either intervention alone.

\section{General rules}

- Look at how others model similar situations adapt their models to the present situation.

- Collect / ask for background information needed to understand the problem.

- Start with simple models; add details as they become known and useful or necessary.

- Find all relevant quantities and make them precise.

- Find all relevant relationships between quantities ( [differential] equations, inequalities, case distinctions ).

- Locate / collect / select the data needed to specify these relationships.

- Find all restrictions that the quantities must obey (sign, limits, forbidden overlaps, etc.). Which restrictions are hard, which soft? How sof $\mathrm{t}$ ?

- Try to incorporate qualitative constraints that rule out otherwise feasible results (usually from inadequate previous versions).

- Find all goals (including conflicting ones)

- Play the devil's advocate to find out and formulate the weak spots of your model.

- Sort available information by the degree of impact expected/hoped for.

- Create a hierarchy of models: from coarse, highly simplifying models to models with all known details. Are there useful toy models with simpler data? Are there limiting cases where the model simplifies? Are there interesting extreme cases that help discover difficulties?

- First solve the coarser models (cheap but inaccurate) to get good starting points for the finer models (expensive to solve but realistic)

- Try to have a simple working model (with report) after $1 / 3$ of the total time planned for the task. Use the remaining time for improving or expanding the model based on your experience, for making the programs more versatile and speeding them up, for polishing documentation, etc.

- Good communication is essential for good applied work.

- The responsibility for understanding, for asking the questions that lead to it, for recognizing misunderstanding (mismatch between answers expected and answers received), and for overcoming them lies with the mathematician. You cannot usually assume your customer to understand your scientific jargon.

- Be not discouraged. Failures inform you about important missing details in your understanding of the problem (or the customer/boss) - utilize this information!

- There are rarely perfect solutions. Modeling is the art of finding a satisfying compromise. Start with the highest standards, and lower them as the deadline approaches. If you have results early, raise your standards again.

- Finish your work in time.

\section{Attitudes}

- Do whatever you do with love. Love (even in difficult circumstances) can be learnt; it noticeably improves the quality of your work and the satisfaction you derive from it.

- Do whatever you do as a service to others. This will improve your attention, the feedback you'll get, and the impact you'll have.

- Take responsibility; ask if in doubt; read to confirm your understanding. This will remove many impasses that otherwise would delay your work.

\section{REFERENCES}

1. Apostolico et al., 2009. Apostolico A., Ciriello G., Guerra C., Heitsch C. E., Hsiao C., Williams L. D. Finding 3D motifs in ribosomal RNA structures. Nucleic Acids Res. 2009; 37:e29.

2. Boaler, 1998. Boaler J. Open and closed mathematics: student experiences and understandings. J. Res. Math. Educ. 1998; 29:4162.

3. Committee on Mathematical Sciences Research for DOEs Computational Biology, National Research Council, 2005. Committee on Mathematical Sciences Research for DOEs Computational Biology, National Research Council. Washington, DC: The National Academies Press; 2005. Mathematics and 21st Century Biology.

4. Committee on Prospering in the Global Economy of the 21st Century: An Agenda for American Science and Technology, 2007. Committee on 
Prospering in the Global Economy of the 21st Century: An Agenda for American Science and Technology. Washington, DC: The National Academies Press; 2007. Rising Above The Gathering Storm: Energizing and Employing America for a Brighter Economic Future.

5. Cox et al., 2007. Cox D., Little J., O'Shea D. 3rd ed. New York: Springer; 2007. Ideals, Varieties, and Algorithms: An Introduction to Computational Algebraic Geometry and Commutative Algebra.

6. Laubenbacher and Stigler, 2004. Laubenbacher R., Stigler B. A computational approach to the reverse engineering of gene regulatory networks. J. Theor. Biol. 2004; 229:523-537.

7. Martins et al., 2008. Martins A. M., Vare-Licona P., Laubenbacher R. Model your genes the mathematical way. Teach. Math. Appl. 2008; 27:91-101.

8. National Research Council, 2005. National Research Council. Mathematics and 21st Century Biology. Washington, DC: National Academies Press; 2005.

9. Robeva, 2009. Robeva R. Desegregating undergraduate mathematics and biology interdisciplinary instruction with emphasis on ongoing research. In: Johnson M. L., Brand L., editors. Methods in Enzymology 454, Computer Methods. Part A. San Diego, CA: Elsevier Academic Press; 2009.

10. Robeva and Laubenbacher, 2009. Robeva R., Laubenbacher R. Mathematical biology education: beyond calculus. Science. 2009; 325:542-543. 